\title{
Integer-valued polynomials over matrices and divided differences
}

\author{
Giulio Peruginelli*
}

January 25, 2020

\begin{abstract}
Let $D$ be an integrally closed domain with quotient field $K$ and $n$ a positive integer. We give a characterization of the polynomials in $K[X]$ which are integer-valued over the set of matrices $M_{n}(D)$ in terms of their divided differences. A necessary and sufficient condition on $f \in K[X]$ to be integer-valued over $M_{n}(D)$ is that, for each $k$ less than $n$, the $k$-th divided difference of $f$ is integral-valued on every subset of the roots of any monic polynomial over $D$ of degree $n$. If in addition the intersection of the maximal ideals of finite index is (0) then it is sufficient to check the above conditions on subsets of the roots of monic irreducible polynomials of degree $n$, that is, conjugate integral elements of degree $n$ over $D \underline{1}$
\end{abstract}

Keywords: Integer-valued polynomial, Divided differences, Matrix, Integral element, Polynomial closure, Pullback. MSC Classification codes: 13B25, 13F20, 11C20.

\section{Introduction}

Let $D$ be a domain with quotient field $K$. We denote by $M_{n}(D)$ the $D$-algebra of $n \times n$ matrices with entries in $D$. We consider the ring of polynomials in $K[X]$ which are integer-valued over $M_{n}(D)$ (see [5], for a general reference), namely:

$$
\operatorname{Int}\left(M_{n}(D)\right) \doteqdot\left\{f \in K[X] \mid f(M) \in M_{n}(D), \forall M \in M_{n}(D)\right\} .
$$

In 3 . the authors consider the overring of integer-valued polynomials which are integer-valued over the subset $T_{n}(D) \subset M_{n}(D)$ of triangular matrices over $D$, namely the $\operatorname{ring} \operatorname{Int}\left(T_{n}(D)\right) \doteqdot$ $\left\{f \in K[X] \mid f(T) \in M_{n}(D), \forall T \in T_{n}(D)\right\}$. They characterize a polynomial $f(X)$ of this ring in

\footnotetext{
*Institut für Analysis und Comput. Number Theory, Technische Univ., Steyrergasse 30, A-8010 Graz, Austria. E-mail: peruginelli@math.tugraz.at.

${ }^{1}$ The only difference from this version and the official one (http://dx.doi.org/10.1007/s00605-013-0519-9) is that the assumption " $D$ has zero Jacobson radical" in (4), (5) and Thm 4.1 has to be replaced with "the intersection of the maximal ideals of finite index is (0)".
} 
terms of its divided differences, proving that up to the order $n-1$ the $k$-th divided difference $\Phi^{k}(f) \in K\left[X_{1}, \ldots, X_{k+1}\right]$ is integer-valued over $D^{k+1}$, that is $\Phi^{k}(f)\left(D^{k+1}\right) \subset D$. See section 4 of this paper for the definition of divided differences of a polynomial.

We give here an analogous characterization for a polynomial $f(X)$ in $\operatorname{Int}\left(M_{n}(D)\right)$. It turns out that, for every $0 \leq k<n, \Phi^{k}(f)$ is integral-valued on every subset of $k+1$ elements of the roots of any monic polynomial $p \in D[X]$ of degree $n$. More precisely, given such a polynomial $p(X)$, let $\alpha_{1}, \ldots, \alpha_{n}$ be its roots in a fixed algebraic closure of $K$, counted with multiplicity. Then $\Phi^{k}(f)\left(\alpha_{1}, \ldots, \alpha_{k+1}\right)$ is integral over $D$, for every possible choice of the subset $\left\{\alpha_{i}\right\}_{i=1}^{k+1}$ of the roots of $p(X)$. This property of $\Phi^{k}(f)$ holds for every polynomial $p(X)$ chosen as before. Notice that the $\alpha_{i}$ 's are integral over $D$ since $p(X)$ is monic (they are not conjugate over $D$, if $p(X)$ is reducible). These properties about the divided differences of $f(X)$ are sufficient and necessary for $f(X)$ to belong to $\operatorname{Int}\left(M_{n}(D)\right)$. This characterization generalizes the previous one about integer-valued polynomials over triangular matrices. In fact, for such a polynomial $f(X)$ and for all the relevant $k$ 's, $\Phi^{k}(f)$ is integer-valued on every subset of $k+1$ roots of every monic polynomial over $D$ of degree $n$ which is totally split over $D$.

Here is an overview of the paper. In the second section we give a characterization of the ring of polynomials which are integer-valued over the set of matrices with prescribed characteristic polynomial: it turns out that this ring has a quite simple algebraic structure, being equal to a pullback of $K[X]$ (Lemma 2.2 and Remark 2.1). In the third section, under the assumption that $D$ is integrally closed, we prove that an ideal in $D[X]$ generated by a non-zero element of $D$ and a non-constant monic polynomial is contracted with respect to any polynomial ring extension $D^{\prime}[X]$, under a suitable assumption. In the fourth section we recall the definition of divided differences and in Theorem 4.1 we use the previous results to characterize integer-valued polynomials over matrices in terms of their divided differences. In the fifth section we give some results about integer-valued polynomials over triangular matrices. In the sixth section we give some results about the so-called polynomial closure of set of matrices having prescribed characteristic polynomial. Finally, in the last section we give some remarks which follows from our main Theorem 4.1

\section{Integer-valued polynomials over matrices with prescribed characteristic polynomial}

Given a commutative ring $R$ and a matrix $M \in M_{n}(R)$, we denote by $p_{M} \in R[X]$ the characteristic polynomial of $M$ and by $N_{R[X]}(M)$ the ideal of polynomials $g(X)$ in $R[X]$ such that $g(M)=0$, also called the null ideal of $M$ over $R$.

As in 3 , given a subset $S$ of $M_{n}(D)$ we consider the ring of polynomials which are integer-valued over $S$ :

$$
\operatorname{Int}\left(S, M_{n}(D)\right) \doteqdot\left\{f \in K[X] \mid f(M) \in M_{n}(D), \forall M \in S\right\} .
$$

Notice that we have the containment $\operatorname{Int}\left(M_{n}(D)\right) \subseteq \operatorname{Int}\left(S, M_{n}(D)\right)$.

We introduce the following definition:

Definition 2.1. For a given positive integer $n$, we denote by $\mathcal{P}_{n}$ the set of monic polynomials of degree $n$ in $D[X]$. Given a subset $P \subseteq \mathcal{P}_{n}$, we denote by

$$
M_{n}^{P}(D) \doteqdot\left\{M \in M_{n}(D) \mid p_{M}(X) \in P\right\}
$$


the set of matrices in $M_{n}(D)$ whose characteristic polynomial $p_{M}(X)$ is in $P$. To ease the notation, given a polynomial $p \in \mathcal{P}_{n}$, we set $M_{n}^{p}(D)=M_{n}^{\{p\}}(D)$.

Notice that $M_{n}^{p}(D)$ is always non-empty, since the companion matrix $C_{p}$ of $p(X)$ belongs to $M_{n}^{p}(D)$. Given $p \in \mathcal{P}_{n}$, we begin to study the corresponding ring of integer-valued polynomials $\operatorname{Int}\left(M_{n}^{p}(D), M_{n}(D)\right)$.

The following Lemma is easy to prove.

Lemma 2.1. Let $R$ be a commutative ring and $p \in R[X]$ a monic polynomial of degree $n$. Then

$$
\bigcap_{M \in M_{n}^{p}(R)} N_{R[X]}(M)=(p(X)) .
$$

Proof : By Cayley-Hamilton theorem we have that $p(X)$ annihilates every matrix in $M_{n}^{p}(R)$. Conversely, we use the fact that over any commutative ring $R$ the null ideal of the companion matrix of a polynomial $p(X)$ is the principal ideal generated by $p(X)$ (see [4). Hence, the above intersection is contained in $N_{R[X]}\left(C_{p}\right)=(p(X))$, so we are done.

The next lemma follows immediately from Lemma 2.1. It gives a characterization of the ring of integer-valued polynomials over the set of matrices with a prescribed characteristic polynomial.

Lemma 2.2. Let $p \in D[X]$ be a monic polynomial of degree $n$ and $f(X)=g(X) / d \in K[X]$, $g \in D[X], d \in D \backslash\{0\}$. Then the following are equivalent:

1) $f \in \operatorname{Int}\left(M_{n}^{p}(D), M_{n}(D)\right)$.

2) $g(X)$ is divisible by $p(X)$ modulo $d D[X]$.

3) the remainder of the division of $f(X)$ by $p(X)$ in $K[X]$ is in $D[X]$, that is:

$$
f(X)=r(X)+p(X) q(X),
$$

for some $q \in K[X], p \in D[X]$ with $\operatorname{deg}(p)<n$.

4) $f \in \operatorname{Int}\left(\left\{C_{p}\right\}, M_{n}(D)\right)$, where $C_{p}$ is the companion matrix of $p(X)$.

Proof : Given $f(X)=g(X) / d \in K[X]$, it is easy to see that (see also [4]) we have

$$
f(M) \in M_{n}(D) \text { if and only if } \bar{g} \in N_{(D / d D)[X]}(\bar{M}),
$$

where $N_{(D / d D)[X]}(\bar{M})$ is the null ideal over $(D / d D)[X]$ of the matrix $\bar{M}$ obtained by reducing the entries of $M$ modulo the ideal $d D$ and $\bar{g}$ is the polynomial obtained by reducing the coefficients of $g$ modulo $d D$. Since $\operatorname{Int}\left(M_{n}^{p}(D), M_{n}(D)\right)=\bigcap_{M \in M_{n}^{p}(D)} \operatorname{Int}\left(\{M\}, M_{n}(D)\right)$, we get

$$
\operatorname{Int}\left(M_{n}^{p}(D), M_{n}(D)\right)=\left\{f(X)=g(X) / d \in K[X] \mid \bar{g} \in \bigcap_{M \in M_{n}^{p}(D)} N_{(D / d D)[X]}(\bar{M})\right\}
$$

(notice that given $g(X) / d \in K[X]$, the reduction on the right is modulo $d$ ). By Lemma 2.1] we get that 1$) \Leftrightarrow 2 \sqrt{2}$.

\footnotetext{
${ }^{2}$ This proof can be shortened using directly the above result that over any commutative ring $R$, the null ideal of the companion matrix of a polynomial $p(X)$ is the principal ideal generated by $p(X)$.
} 
Since the polynomial $p(X)$ is monic, by the Euclidean division algorithm condition 2 ) is equivalent to the condition that $g(X)$ belongs to the ideal of $D[X]$ generated by $p(X)$ and $d$. Clearly, this is equivalent to condition 3$)$.

Finally, by the aforementioned result about null ideals of companion matrices, we have

$$
\operatorname{Int}\left(M_{n}^{p}(D), M_{n}(D)\right)=\operatorname{Int}\left(\left\{C_{p}\right\}, M_{n}(D)\right) .
$$

Remark 2.1. We notice that condition 3) says that

$$
\operatorname{Int}\left(M_{n}^{p}(D), M_{n}(D)\right)=D[X]+p(X) \cdot K[X]
$$

where the latter ring is a pullback of $K[X]$. In particular, a polynomial $f \in K[X]$ of degree less than $n$ is integer-valued over $M_{n}^{p}(D)$ if and only if $f(X)$ is in $D[X]$.

It is easy to generalize Lemma 2.2 to integer-valued polynomials over $M_{n}^{P}(D)$, for a given subset $P \subseteq \mathcal{P}_{n}$. Since $M_{n}^{P}(D)=\bigcup_{p \in P} M_{n}^{p}(D)$, we have

$$
\operatorname{Int}\left(M_{n}^{P}(D), M_{n}(D)\right)=\bigcap_{p \in P} \operatorname{Int}\left(M_{n}^{p}(D), M_{n}(D)\right) .
$$

The following Proposition follows by Lemma 2.2 and the previous representation of the ring $\operatorname{Int}\left(M_{n}^{P}(D), M_{n}(D)\right)$ as an intersection of the overrings $\operatorname{Int}\left(M_{n}^{p}(D), M_{n}(D)\right)$.

Proposition 2.1. Let $P$ be a set of monic polynomials in $D[X]$ of degree $n$. Let $f(X)=g(X) / d \in$ $K[X]$. Then

$$
f \in \operatorname{Int}\left(M_{n}^{P}(D), M_{n}(D)\right) \Leftrightarrow g(X) \text { is divisible modulo } d D[X] \text { by all } p \in P .
$$

Remark 2.2. By [4, Lemma 3.4] a polynomial $f(X)=g(X) / d \in K[X]$ is integer-valued over $M_{n}(D)$ if and only if $g(X)$ is divisible modulo $d D[X]$ by all monic polynomials $p \in D[X]$ of degree $n$. Then by Lemma 2.2 we have this representation of the $\operatorname{ring} \operatorname{Int}\left(M_{n}(D)\right)$ :

$$
\operatorname{Int}\left(M_{n}(D)\right)=\bigcap_{p \in \mathcal{P}_{n}} \operatorname{Int}\left(M_{n}^{p}(D), M_{n}(D)\right)
$$

Notice that this is Proposition 2.1 for $P=\mathcal{P}_{n}$.

Suppose now that the intersection of the maximal ideals of finite index is (0). By Proposition 6.2 of $[5]^{3}$ we can just check the previous condition on irreducible polynomials; namely, a polynomial $f(X)=g(X) / d \in K[X]$ is integer-valued over $M_{n}(D)$ if and only if $g(X)$ is divisible modulo $d D[X]$ by all monic irreducible polynomials $p \in D[X]$ of degree $n$. For a given integer $n$, we denote by $\mathcal{P}_{n}^{\text {irr }}$ the set of monic and irreducible polynomials in $D[X]$ of degree $n$. We consider the set of matrices $M_{n}^{\mathrm{irr}}(D) \subsetneq M_{n}(D)$ with irreducible characteristic polynomial. Then the last result shows that

$$
\operatorname{Int}\left(M_{n}(D)\right)=\operatorname{Int}\left(M_{n}^{\mathrm{irr}}(D), M_{n}(D)\right)
$$

\footnotetext{
${ }^{3}$ We notice that in [5. Proposition 6.2] the assumption " $D$ has zero Jacobson radical" has to be replaced with "the intersection of the maximal ideals of finite index is (0)". Indeed, that theorem is clearly false if for example $K$ is algebraically closed.
} 
that is, $M_{n}^{\mathrm{irr}}(D)$ is polynomially dense in $M_{n}(D)$ (see $[3]$ for the definition, which we recall later in section 6). Hence, in this case, since $M_{n}^{\text {irr }}(D)=\bigcup_{p \in \mathcal{P}_{n}^{\text {irr }}} M_{n}^{p}(D)$ we have:

$$
\operatorname{Int}\left(M_{n}(D)\right)=\bigcap_{p \in \mathcal{P}_{n}^{\text {irr }}} \operatorname{Int}\left(M_{n}^{p}(D), M_{n}(D)\right)
$$

\section{A contracted ideal in a polynomial ring extension}

The next lemma says the following. Let $p(X)$ a monic polynomial over $D$. If a polynomial $g \in D[X]$ is divisible by $p(X)$ modulo an element $d$ of $D$ over a ring extension $D^{\prime}$ of $D$ (under a suitable hypothesis), then the same modular divisibility condition for $g(X)$ already holds over $D$ itself. Notice that this is exactly condition 2) of Lemma 2.2.

Lemma 3.1. Let $D \subset D^{\prime}$ be an extension of domains with quotient fields $K \subset K^{\prime}$, respectively, such that $D^{\prime} \cap K=D$. Let $p \in D[X]$ be monic and $d \in D$. Let $g \in D[X]$ be such that $g(X)$ is divisible in $D^{\prime}[X]$ by $p(X)$ modulo $d D^{\prime}[X]$. Then $g(X)$ is divisible in $D[X]$ by $p(X)$ modulo $d D[X]$.

Remark 3.1. Equivalently, the lemma says that the ideal of $D[X]$ generated by $d$ and $p(X)$ is a contracted ideal with respect to $D^{\prime}[X]$, that is:

$$
d D[X]+p(X) D[X]=\left(d D^{\prime}[X]+p(X) D^{\prime}[X]\right) \cap D[X] .
$$

We also notice that if $d=0$ we have $p(X) D[X]=p(X) D^{\prime}[X] \cap D[X]$ for every extension of domains $D \subset D^{\prime}$. In this case we only use the assumption that $p(X)$ is monic.

Proof : As already observed in [6], the equality $D^{\prime} \cap K=D$ is equivalent to the condition that every principal ideal of $D$ is contracted with respect to $D^{\prime}$, that is $d D^{\prime} \cap D=d D$, for every $d \in D$ (the proof is straightforward). From this fact it easily follows that $d D^{\prime}[X] \cap D[X]=d D[X]$.

Let now $g \in D[X]$ be such that $g \in d D^{\prime}[X]+p(X) D^{\prime}[X]$. Then

$$
g(X)=Q(X) p(X)+d R(X)
$$

for some $Q, R \in D^{\prime}[X]$. By Euclidean division $(p(X)$ is monic) we can assume that $\operatorname{deg}(R)<\operatorname{deg}(p)$ (if not, just divide $R(X)$ by $p(X)$ in $\left.D^{\prime}[X]\right)$. We divide now $g(X)$ by $p(X)$ in $D[X]$ and we get

$$
g(X)=q(X) p(X)+r(X)
$$

for some $q, r \in D[X], \operatorname{deg}(r)<\operatorname{deg}(p)$. By the uniqueness of quotient and remainder in $D^{\prime}[X]$ we have $Q(X)=q(X)$ and $d R(X)=r(X)$. This implies that $Q \in D[X]$. By the remark at the beginning of this proof, the second equality implies that $R \in D[X]$, and this concludes the proof.

Example 3.1. In general the statement of Lemma 3.1 is false, if we drop the assumption $D^{\prime} \cap K=$ $D$. For example, let $D=\mathbb{Z}[\sqrt{5}] \subsetneq D^{\prime}=O_{K}=\mathbb{Z}[(1+\sqrt{5}) / 2] \subset K=\mathbb{Q}(\sqrt{5})$, where $O_{K}$ is the ring of integers of the number field $K\left(O_{K}\right.$ is also equal to the integral closure of $D$ in $\left.K\right)$. Obviously, 
$D$ and $D^{\prime}$ have the same quotient field $K$ and $D^{\prime} \cap K \supsetneq D$. We have $2 D \subsetneq 2 O_{K} \cap D=2 O_{K} \subset D$ (last equality holds because $2 O_{K}$ is the conductor of the integral extension $D \subset O_{K}$ ). The element $\alpha=1+\sqrt{5}$ is in $2 O_{K}$ but not in $2 D$, so that the polynomial $g(X)=X+\alpha \in D[X]$ is divisible in $O_{K}[X]$ by $X$ modulo $2 O_{K}[X]$ but it is not divisible in $D[X]$ by $X$ modulo $2 D[X]$.

Remark 3.2. The Lemma 3.1 applies in the following settings: let $D$ be integrally closed, $E$ any field extension of $K$ and $D_{E}$ the integral closure of $D$ in $E$. Then $D_{E} \cap K=D$, so the above statement holds with $D_{E}$ at the place of $D^{\prime}$. Under these conditions, given a monic polynomial $p \in D[X]$ of degree $n$, Lemma 2.2 and 3.1 together show that

$$
\operatorname{Int}\left(M_{n}^{p}\left(D_{E}\right), M_{n}\left(D_{E}\right)\right) \cap K[X]=\operatorname{Int}\left(M_{n}^{p}(D), M_{n}(D)\right) .
$$

In particular, if we extend the field $K$ to the splitting field $F$ of $p(X)$, then by Lemma 3.1 the same modular divisibility relation for a polynomial $g \in D[X]$ of condition 2) of Lemma 2.2 holds. In this way, in the next section we will be able to apply the Newton expansion of a polynomial $g \in D[X]$ with respect to the set of roots of $p(X)$ in $D_{F}$ (the coefficients of this expansion are exactly the divided differences of $g(X)$ evaluated at the roots of $p(X))$.

\section{Divided differences of integer-valued polynomials over ma- trices}

Let $g(X)$ be a polynomial of degree $n$ over a commutative ring $R$ and let $a_{0}, \ldots, a_{n}$ be arbitrary chosen elements of $R$ (possibly with repetitions).

We have the following formula due to Newton:

$$
\begin{array}{r}
g(X)=g\left(a_{0}\right)+\Phi^{1}(g)\left(a_{0}, a_{1}\right)\left(X-a_{0}\right)+\Phi^{2}(g)\left(a_{0}, a_{1}, a_{2}\right)\left(X-a_{0}\right)\left(X-a_{1}\right)+\ldots \\
+\Phi^{n}(g)\left(a_{0}, \ldots, a_{n}\right)\left(X-a_{0}\right) \cdot \ldots \cdot\left(X-a_{n-1}\right)
\end{array}
$$

where, for each $k \in \mathbb{N}, \Phi^{k}(g)\left(X_{0}, \ldots, X_{k}\right)$ is the $k$-th divided difference of $g$ defined recursively as follows:

$$
\begin{aligned}
\Phi^{0}(g)\left(X_{0}\right) & \doteqdot g(X) \\
\Phi^{1}(g)\left(X_{0}, X_{1}\right) & \doteqdot \frac{g\left(X_{0}\right)-g\left(X_{1}\right)}{X_{0}-X_{1}} \\
& \ldots \\
\Phi^{k}(g)\left(X_{0}, \ldots, X_{k}\right) & \doteqdot \frac{\Phi^{k-1}(g)\left(X_{0}, \ldots, X_{k-1}\right)-\Phi^{k-1}(g)\left(X_{0}, \ldots, X_{k-2}, X_{k}\right)}{X_{k-1}-X_{k}}
\end{aligned}
$$

$\Phi^{k}(g)$ is a symmetric polynomial with coefficients in $R$ in $k+1$ variables. Obviously, a polynomial $g \in R[X]$ is divisible by $\left(X-a_{0}\right) \cdot \ldots \cdot\left(X-a_{n-1}\right)$ if and only if the coefficients $\Phi^{k}(g)\left(a_{0}, \ldots, a_{k}\right)$ of the expansion in (6) are equal to zero, for all $0 \leq k<n$. We just use the fact that $\{1\} \cup$ $\left\{\prod_{i=0, \ldots, k}\left(X-a_{i}\right)\right\}_{k=0, \ldots, n-1}$ are linearly independent over $D$. More in general, if $\left\{a_{i}\right\}_{i \in \mathbb{N}}$ is a sequence in $R$, then $\{1\} \cup\left\{\prod_{i=0, \ldots, k}\left(X-a_{i}\right)\right\}_{k \in \mathbb{N}}$ is a free basis of the $R$-module $R[X]$. 
The next lemma appears also in [3, Proposition 11], but for the sake of the reader we report it here.

Lemma 4.1 (3]). Let $D$ be a domain. Let $g \in D[X], d \in D \backslash\{0\}$ and $a_{0}, \ldots, a_{n} \in D$ (not necessarily distinct). Then $g(X)$ is divisible modulo $d D[X]$ by $p(X)=\prod_{i=0, \ldots, n}\left(X-a_{i}\right)$ if and only if for all $0 \leq k<n$ we have $\Phi^{k}(g)\left(a_{0}, \ldots, a_{k}\right) \in d D$.

Proof : Consider everything modulo $d D$ and apply Newton's formula.

Let $D$ be an integrally closed domain with quotient field $K$. Remember that for any extension $E$ of $K$ we denote by $D_{E}$ the integral closure of $D$ in $E$. From now on we consider only algebraic extension of $K$, which are tacitly assumed to be contained in $\bar{K}$. We recall that a multi-set is a set with repetitions. For instance, the multi-set of roots of a polynomial $p \in K[X]$ is the multi-set formed by the roots of the polynomial in $\bar{K}$ each root being repeated a number of times equals to its multiplicity in $p(X)$. We give now the following proposition:

Proposition 4.1. Let $D$ be an integrally closed domain with quotient field $K$. Let $g \in D[X]$, $d \in D \backslash\{0\}$ and $p \in D[X]$ monic of degree $n$. Let $\Omega_{p}=\left\{\alpha_{1}, \ldots, \alpha_{n}\right\}$ be the multi-set of roots of $p(X)$ in a splitting field $F$ over $K$. Then $f(X)=g(X) / d \in \operatorname{Int}\left(M_{n}^{p}(D), M_{n}(D)\right)$ if and only if for all $0 \leq k<n, \Phi^{k}(g)\left(\alpha_{i_{0}}, \ldots, \alpha_{i_{k}}\right) \in d D_{F}$, for all subsets $\left\{i_{0}, \ldots, i_{k}\right\}$ of $k+1$ elements of $\{1, \ldots, n\}$.

Proof : Suppose $f(X)=g(X) / d$, for some $g \in D[X]$ and $d \in D, d \neq 0$. By Lemma 2.2, $f(X)$ is in $\operatorname{Int}\left(M_{n}^{p}(D), M_{n}(D)\right)$ if and only if $g(X)$ is divisible modulo $d D[X]$ by $p(X)$. Since $D$ is integrally closed, by Lemma 3.1 this is equivalent to $g(X)$ divisible modulo $d D_{F}[X]$ by $p(X)$. If we fix an order of the roots $\alpha_{1}, \ldots, \alpha_{n}$ of $p(X)$, by Newton's formula (6) and Lemma 4.1, the previous condition holds if and only if $\Phi^{k}(g)\left(\alpha_{0}, \ldots, \alpha_{k}\right) \in d D_{F}$ for all $0 \leq k<n$. Since this holds for every possible order of the $\alpha_{i}$ 's we may choose (because the above modular divisibility condition does not depend on the order of the roots $\Omega_{p}$ ), we get the final statement.

Remark 4.1. Notice that the roots of $p(X)$ are integral over $D$ (hence they are in $D_{F}$ ) and by definition $F=K\left(\alpha_{1}, \ldots, \alpha_{n}\right)$. In particular, for each of the relevant $k, \Phi^{k}(f)\left(\alpha_{i_{0}}, \ldots, \alpha_{i_{k}}\right) \in D_{F_{k}}$, where $F_{k}$ is the field generated by $\alpha_{i_{0}}, \ldots, \alpha_{i_{k}}$ over $K$. We stress that we are not assuming $p(X)$ to be irreducible. Notice also that the above condition " $\Phi^{k}(g)\left(\alpha_{i_{0}}, \ldots, \alpha_{i_{k}}\right) \in d D_{F}$ " is equivalent to $" \Phi^{k}(f)\left(\alpha_{i_{0}}, \ldots, \alpha_{i_{k}}\right)$ is integral over $D "$ (since, obviously, $\Phi^{k}(f)\left(\alpha_{i_{0}}, \ldots, \alpha_{i_{k}}\right)$ belongs to $F$ ). If the conditions on the values of the divided differences $\Phi^{k}(g)$, for $0 \leq k<n$, at the roots $\Omega_{p}$ of $p(X)$ hold for a fixed ordering, then they hold for every other order we may choose.

Example 4.1. Let us see with an example what happens in the case $n=2$. Let $p \in D[X]$ be an irreducible monic polynomial of degree 2. Let $\alpha$ be a root of $p(X)$ in $\bar{K}$. Over the quadratic extension $K(\alpha)$ of $K$ we have $p(X)=(X-\alpha)\left(X-\alpha^{\prime}\right)$, where $\alpha^{\prime}$ is the conjugate root of $\alpha$. Then, given a polynomial $f(X)$ in $K[X], f(X)$ is integer-valued over the set of matrices in $M_{2}(D)$ with characteristic polynomial equal to $p(X)$ if and only if $f(\alpha)$ and $\Phi^{1}(f)\left(\alpha, \alpha^{\prime}\right)$ are integral over $D$ (by above, these two conditions implies that also $f\left(\alpha^{\prime}\right)$ is integral over $\left.D\right)$. If the extension $K(\alpha) \supset K$ is separable, then $\Phi^{1}(f)\left(\alpha, \alpha^{\prime}\right) \in D$, because $\Phi^{1}(f)(X, Y)$ is symmetric, so in particular $\Phi^{1}(f)\left(\alpha, \alpha^{\prime}\right)$ is invariant under the action of the Galois group of $K(\alpha)$ over $K$. If we consider a split polynomial 
$p(X)$ in $D[X]$, say $p(X)=(X-a)(X-b)$, for some $a, b \in D$, then $f \in \operatorname{Int}\left(M_{2}^{p}(D), M_{2}(D)\right)$ if and only if $f(a)$ and $\Phi^{1}(f)(a, b)$ are in $D$.

Finally, by Proposition 4.1 and the representation of $\operatorname{Int}\left(M_{n}(D)\right)$ in (3) and (5), we give this characterization of the polynomials of $\operatorname{Int}\left(M_{n}(D)\right)$ in terms of their divided differences.

Theorem 4.1. Let $D$ be an integrally closed domain. Let $f \in K[X]$ and $n$ a positive integer. Then $f \in \operatorname{Int}\left(M_{n}(D)\right)$ if and only if for every $0 \leq k<n$, for every monic polynomial $p \in D[X]$ of degree $n$ and for every multi-set of $k+1$ elements $\left\{\alpha_{1}, \ldots, \alpha_{k+1}\right\}$ of the roots of $p(X)$ in $\bar{K}$, $\Phi^{k}(f)\left(\alpha_{1}, \ldots, \alpha_{k+1}\right)$ is integral over $D$.

If the intersection of the maximal ideals of finite index is (0) then it is sufficient to consider polynomials $p \in D[X]$ which are irreducible.

Proof : By representation (3) and Proposition 4.1 $f \in \operatorname{Int}\left(M_{n}(D)\right)$ if and only if for all $0 \leq k<n$, $\Phi^{k}(f)\left(\alpha_{i_{0}}, \ldots, \alpha_{i_{k}}\right)$ is integral over $D$ for every multi-set of $k+1$ elements of the roots $\Omega_{p}$ of any $p \in$ $\mathcal{P}_{n}$. Notice that, by the arguments given in the proof of Proposition 4.1] and the Remark 4.1, if for every $p \in \mathcal{P}_{n}$ we fix an ordering of the roots $\Omega_{p}$ of $p(X)$, for $f(X)$ to belong to $\operatorname{Int}\left(M_{n}^{p}(D), M_{n}(D)\right)$ it is sufficient that each $k$-th divided difference of $f(X)$ is integral on the first $k+1$ roots of $p(X)$. The last claim follows by (5).

Remark 4.2. The roots of any $p \in \mathcal{P}_{n}$ have degree over $K$ bounded by $n$. Notice that the divided differences $\Phi^{k}(f)$, for $0 \leq k<n$, of an integer-valued polynomial $f(X)$ over $M_{n}(D)$ are integral on multi-sets $\left\{\alpha_{1}, \ldots, \alpha_{k+1}\right\}$ of integral elements over $D$ which are roots of polynomials in $\mathcal{P}_{n}$, that is, the elements $\left\{\alpha_{1}, \ldots, \alpha_{k+1}\right\}$ cannot be chosen independently (except when their degree is one, that is, they lie in $D$; this happens if $p(X)$ is totally split over $D)$. Moreover, if the intersection of the maximal ideals of finite index is (0), we may just consider polynomials in $\mathcal{P}_{n}^{\text {irr }}$, so that the $k$-th divided differences of $f(X)$ are integral on every set of $k+1$ conjugate integral elements of degree $n$ over $D$ inside $\bar{K}$.

Example 4.2. Here we continue the Example 4.1] A polynomial $f \in K[X]$ is integer-valued over $M_{2}(D)$ if and only if the following two conditions hold:

i) for every $\alpha \in \bar{K}$, integral over $D$ of degree $2, f(\alpha)$ and $\Phi^{1}(f)\left(\alpha, \alpha^{\prime}\right)$ are integral over $D$ (where as before $\alpha^{\prime}$ is the conjugate of $\alpha$ over $K$ ).

ii) for every $(a, b) \in D^{2}, f(a), \Phi^{1}(f)(a, b)$ are in $D$.

If the intersection of the maximal ideals of finite index is $(0)$, then $f \in \operatorname{Int}\left(M_{2}(D)\right)$ if and only if condition i) holds.

\section{Integer-valued polynomials over triangular matrices with prescribed characteristic polynomial}

We give here some characterizations of integer-valued polynomials over triangular matrices. Now $D$ is again just an integral domain (that is, not necessarily integrally closed). The following definition is similar to Definition 2.1. 
Definition 5.1. Let $\mathcal{P}_{n}^{s}$ be the set of monic polynomials in $D[X]$ of degree $n$ which are totally split in $D$. Given a subset $P \subset \mathcal{P}_{n}^{s}$ we denote by

$$
T_{n}^{P}(D) \doteqdot\left\{T \in T_{n}(D) \mid p_{T}(X) \in P\right\}
$$

the set of triangular matrices whose characteristic polynomial is in $P$. For a single polynomial $p \in \mathcal{P}_{n}^{s}$, we set $T_{n}^{p}(D)=T_{n}^{\{p\}}(D)$.

The next lemma is analogous to [3, Proposition 15].

Lemma 5.1. Let $p \in D[X]$ be a totally split polynomial of degree $n$. Let $f(X)=g(X) / d$ be in $K[X]$. Then

$$
f \in \operatorname{Int}\left(T_{n}^{p}(D), M_{n}(D)\right) \Leftrightarrow g(X) \text { is divisible by } p(X) \text { modulo } d D[X] .
$$

In particular,

$$
\operatorname{Int}\left(T_{n}^{p}(D), M_{n}(D)\right)=\operatorname{Int}\left(M_{n}^{p}(D), M_{n}(D)\right) .
$$

Proof : One direction is easy (the proof is exactly like in Lemma 2.2): suppose $g(X)=h(X) p(X)+$ $d r(X)$, for some $h, r \in D[X]$. Let $T$ be a triangular matrix with characteristic polynomial equal to $p(X)$. Then by the Cayley-Hamilton theorem we have $g(T)=d r(T)$, so that $f(T)=r(T) \in M_{n}(D)$.

For the converse, we use an inductive argument on the degree of $p(X)$ as in 3, Proposition 14]. Let $n=2$ and let $p(X)=\left(X-a_{0}\right)\left(X-a_{1}\right)$, for some $a_{0}, a_{1} \in D$. Given $f(X)=g(X) / d$ in $\operatorname{Int}\left(T_{2}^{p}(D), M_{2}(D)\right)$, we divide $g(X)$ by $p(X)$ in $D[X]: g(X)=q(X) p(X)+r(X)$, with $q, r \in D[X]$, $\operatorname{deg}(r) \leq 1$, say $r(X)=a X+b$. Take

$$
T=\left(\begin{array}{cc}
a_{0} & 0 \\
1 & a_{1}
\end{array}\right)
$$

Then $g(T)=a T+b \in T_{2}(d D)$ (essentially by (10)) is the triangular matrix

$$
\left(\begin{array}{cc}
a a_{0}+b & 0 \\
a & a a_{1}+b
\end{array}\right)
$$

so that $a, b \in d D$ as wanted.

Suppose now the statement is true up to $n$ and suppose that $p(X)=\prod_{i=0, \ldots, n}\left(X-a_{i}\right)$. Let $p_{1}(X)=\prod_{i=1, \ldots, n}\left(X-a_{i}\right)$. Given $T \in T_{n}^{p_{1}}(D)$, we consider the following $(n+1) \times(n+1)$ matrix:

$$
T_{1}=\left(\begin{array}{c|c} 
& 0 \\
\mathrm{~T} & \vdots \\
& 0 \\
\hline 0 \cdots 0 & a_{0}
\end{array}\right) .
$$

Notice that $T_{1}$ is in $T_{n+1}^{p}(D)$ and it is a block matrix. Let $f \in \operatorname{Int}\left(T_{n+1}^{p}(D), M_{n+1}(D)\right)$. Then $f(X)$ is integer-valued on $T$. In fact,

$$
f\left(T_{1}\right)=\left(\begin{array}{c|c}
\mathrm{f}(\mathrm{T}) & 0 \\
& 0 \\
\hline 0 \cdots 0 & f\left(a_{0}\right)
\end{array}\right)
$$


$f\left(T_{1}\right)$ has entries in $D$, so that $f(T)$ has entries in $D$, too. Since this holds for every $T$ in $T_{n}^{p_{1}}(D)$ and for every $f \in \operatorname{Int}\left(T_{n+1}^{p}(D), M_{n+1}(D)\right)$, we have $\operatorname{Int}\left(T_{n+1}^{p}(D), M_{n+1}(D)\right) \subset \operatorname{Int}\left(T_{n}^{p_{1}}(D), M_{n}(D)\right)$.

By induction hypothesis, given $f(X)=g(X) / d \in \operatorname{Int}\left(T_{n+1}^{p}(D), M_{n+1}(D)\right)$, we have that $g(X)$ is divisible by $p_{1}(X)$ modulo $d D[X]$. Then we continue as in the proof of Proposition 14 of [3], essentially going through the characterization of Lemma 4.1 together with Corollary 13 of 3 .

The last claim follows from Lemma 2.2.

The following proposition follows from the obvious fact $T_{n}^{S}(D)=\bigcup_{p \in S} T_{n}^{p}(D)$. This is analogous to Proposition 2.1.

Proposition 5.1. Let $P$ be a set of monic totally split polynomials in $D[X]$ of degree $n$. Let $f(X)=g(X) / d \in K[X]$. Then

$$
f \in \operatorname{Int}\left(T_{n}^{P}(D), M_{n}(D)\right) \Leftrightarrow g(X) \text { divisible modulo } d D[X] \text { by all } p \in P .
$$

In particular,

$$
\operatorname{Int}\left(T_{n}^{P}(D), M_{n}(D)\right)=\operatorname{Int}\left(M_{n}^{P}(D), M_{n}(D)\right) .
$$

The last statement follows from Proposition 2.1.

\section{Polynomial closure of set of matrices}

We recall from [3] the following definition. Given a subset $S$ in $M_{n}(D)$, the polynomial closure of $S$ in $M_{n}(D)$ is the set

$$
\bar{S} \doteqdot\left\{M \in M_{n}(D) \mid f(M) \in M_{n}(D), \forall f \in \operatorname{Int}\left(S, M_{n}(D)\right)\right\}
$$

$\bar{S}$ is the largest set in $M_{n}(D)$ containing $S$ such that $\operatorname{Int}\left(S, M_{n}(D)\right)=\operatorname{Int}\left(\bar{S}, M_{n}(D)\right)$. A set of matrices $S$ is said to be polynomially closed if $S=\bar{S}$, polynomially dense if $\bar{S}=M_{n}(D)$ (for example, $M_{n}^{\text {irr }}(D)$ is polynomially dense in $M_{n}(D)$, if the intersection of the maximal ideals of finite index is (0), see (4)) .

For some domains $D$, the authors of [3] prove that the polynomial closure of $T_{n}(D)$ is equal to the set $S_{n}(D)$ of all matrices in $M_{n}(D)$ with totally split characteristic polynomial (see [3, Theorem 27]). On the other hand, Proposition 5.1 shows that, for any given subset $P \subseteq \mathcal{P}_{n}^{s}, T_{n}^{P}(D)$ and $M_{n}^{P}(D)$ have the same polynomial closure in $M_{n}(D)$, where $D$ is any integral domain.

Under a rather general assumption, we characterize the polynomial closure $\overline{M_{n}^{p}(D)}$ of $M_{n}^{p}(D)$ in $M_{n}(D)$, for any given monic polynomial $p \in D[X]$ of degree $n$.

Proposition 6.1. Suppose $D$ is an integral domain with zero Jacobson radical. Let $p \in \mathcal{P}_{n}$. Then

$$
\overline{M_{n}^{p}(D)}=\left\{M \in M_{n}(D) \mid p(M)=0\right\} .
$$

Moreover, the eigenvalues (in $\bar{K}$ ) of every $M \in \overline{M_{n}^{p}(D)}$ are roots of $p(X)$.

Proof : By Lemma 2.2 a polynomial $f(X)$ in $\operatorname{Int}\left(M_{n}^{p}(D), M_{n}(D)\right)$ has the form $f(X)=r(X)+$ $p(X) q(X)$, for some $q \in K[X], r \in D[X], \operatorname{deg}(r)<\operatorname{deg}(p)$. Clearly, if $p(M)=0$ then for every 
polynomial $f(X)$ as above, we have $f(M)=r(M) \in M_{n}(D)$. Conversely, a matrix $M$ is in the polynomial closure of $M_{n}^{p}(D)$ if and only if, for every $d \in D \backslash\{0\}$ and for every $g \in D[X]$, we have $p(M) g(M) \in M_{n}(d D)$. In particular (choose $g(X)=1$ ), this implies that $p(M)$ has entries which lie in $\cap_{d \in D \backslash\{0\}} d D$. Since $D$ has zero Jacobson radical, we have $p(M)=0$, as wanted.

For the last claim, let $M$ be a matrix such tht $p(M)=0$. By a result of McCoy (see Corollary p. 283 of [8]) the ideals $N_{D[X]}(M)$ and $\left(p_{M}(X)\right)$ of $D[X]$ have the same radical, so that $p(X)^{m} \in$ $\left(p_{M}(X)\right)$ for some $m \in \mathbb{N}$. In particular, for all the roots $\alpha$ of $p_{M}(X)$ we have $p(\alpha)=0$.

Without the above assumption on $D$, the polynomial closure of $M_{n}^{p}(D)$ is equal to the set $\{M \in$ $\left.M_{n}(D) \mid p(M) \in M_{n}(\mathcal{P})\right\}$, where $\mathcal{P}=\cap_{d \in D \backslash\{0\}} d D$.

Corollary 6.1. Let $D$ be an integral domain with zero Jacobson radical. Let $p \in \mathcal{P}_{n}$ be such that $p(X)$ is irreducible in $K[X]$. Then $M_{n}^{p}(D)$ is polynomially closed.

If Gauss's lemma applies (or just if $D$ is integrally closed, since $p(X)$ is monic), we may just suppose that $p(X)$ is irreducible in $D[X]$.

Proof : Let $M \in M_{n}(D)$ be a matrix in the polynomial closure of $M_{n}^{p}(D)$. By Lemma 6.1 $p(M)=0$. Since $p(X)$ is irreducible it is equal to the minimal polynomial of $M$ (when consired in $M_{n}(K)$; a matrix over a commutative ring $R$ may not have a minimal polynomial). Then the characteristic polynomial of $M$ is equal to the minimal polynomial of $M$, because they always have the same irreducible factors. This means that $M$ is in $M_{n}^{p}(D)$.

We notice that the above argument shows that $M_{n}^{p}(D)=\left\{M \in M_{n}(D) \mid p(M)=0\right\}$, under only the assumption that $p(X)$ is irreducible in $K[X]$ (thus, we don't need for this equality the fact that $D$ has zero Jacobson radical).

We leave the following open problem.

Problem: given any subset $S$ in $\mathcal{P}_{n}$, compute $\overline{M_{n}^{S}(D)}$.

Since $M_{n}^{S}(D)=\bigcup_{p \in S} M_{n}^{p}(D)$, by Lemma 6.1 and known properties of polynomial closure (see [2]) we have

$$
\bigcup_{p \in S} \overline{M_{n}^{p}(D)}=\left\{M \in M_{n}(D) \mid p(M)=0, \text { for some } p \in S\right\} \subset \overline{M_{n}^{S}(D)}
$$

Example 6.1. It is not true in general that the above containment is an equality. Let $D=\mathbb{Z}$ and let $K$ be a quadratic number field with ring of integers $O_{K}$. Let $M_{2}^{K}(\mathbb{Z})$ be the set of matrices in $M_{2}(\mathbb{Z})$ whose characteristic polynomial is the minimal polynomial of some $\alpha \in O_{K} \backslash \mathbb{Z}$. Then by Theorem 4.1 of $\left[9\right.$ we have $\operatorname{Int}\left(M_{2}^{K}(\mathbb{Z}), M_{2}(\mathbb{Z})\right) \subset \operatorname{Int}_{\mathbb{Q}}\left(O_{K}\right) \subset \operatorname{Int}(\mathbb{Z})$, where $\operatorname{Int}_{\mathbb{Q}}\left(O_{K}\right) \doteqdot \operatorname{Int}\left(O_{K}\right) \cap \mathbb{Q}[X]$. In particular this implies that any $2 \times 2$ diagonal matrix $D$ with rational integers in the diagonal is contained in the polynomial closure of $M_{2}^{K}(\mathbb{Z})$. Obviously, the null ideal of such a matrix $D$ over $\mathbb{Z}$ is generated by a totally split polynomial in $\mathbb{Z}$, which is not the minimal polynomial of some quadratic algebraic integer of $K$. 


\section{$7 \quad$ Final Remarks}

Let $f \in \operatorname{Int}\left(M_{n}(D)\right)$. As we anticipated in the introduction, for every $0 \leq k<n, \Phi^{k}(f)$ is integervalued on every multi-set $\left\{\alpha_{0}, \ldots, \alpha_{k}\right\}$ of elements of $D$ : it is sufficient to consider polynomials which split into linear factors in $D[X]$ in Theorem 4.1 This is exactly the reason why our result generalizes [3, Theorem 16]. This implies that $\operatorname{Int}\left(M_{n}(D)\right)$ is a subring of the ring of integer-valued polynomials whose $k$-th divided differences are also integer-valued for all $k<n$. This ring has been introduced by Bharghava in [1] and it is denoted by:

$$
\operatorname{Int}^{\{n-1\}}(D) \doteqdot\left\{f \in K[X] \mid \Phi^{k}(f)\left(D^{k+1}\right) \subset D, \forall 0 \leq k<n\right\} .
$$

By [3, Theorem 16] the latter ring is equal to the ring of polynomials which are integer-valued over the set $T_{n}(D)$ of triangular matrices over $D$.

By the same Theorem 4.1, for $k=0$, we see that $\Phi^{0}(f)(X)=f(X)$ takes integral values on every element $\alpha$ which is integral over $D$ of degree $\leq n$. In particular, $f \in \operatorname{Int}_{K}\left(D_{E}\right) \doteqdot K[X] \cap \operatorname{Int}\left(D_{E}\right)$, for every finite extension $E$ of $K$ of degree $\leq n$ (as usual, $\operatorname{Int}\left(D_{E}\right)$ is the subring of $E[X]$ of those polynomials $f(X)$ such that $\left.f\left(D_{E}\right) \subset D_{E}\right)$. In this way we have the following containment:

$$
\operatorname{Int}\left(M_{n}(D)\right) \subset \bigcap_{[E: K] \leq n} \operatorname{Int}_{K}\left(D_{E}\right) .
$$

In the case $D=\mathbb{Z}$ this inclusion was showed in [7. It can be easily proved that the latter intersection is equal to the $\operatorname{ring} \operatorname{Int}_{K}\left(\mathcal{A}_{n}\right) \doteqdot\left\{f \in K[X] \mid f\left(\mathcal{A}_{n}\right) \subset \mathcal{A}_{n}\right\}$, where $\mathcal{A}_{n}$ is the set of all the integral elements over $D$ in $\bar{K}$ of degree bounded by $n$ (see also [9], in the case $D=\mathbb{Z}$ ).

\section{Acknowledgments}

I wish to thank Keith Johnson for the useful suggestions. I also thank the referee for the several suggestions he/she gave which improved the overall quality of the paper. The author was supported by the Austrian Science Foundation (FWF), Project Number P23245-N18.

\section{References}

[1] M. Bhargava, On P-orderings, Rings of Integer-Valued Polynomials and Ultrametric Analysis, Journal of the Amer. Math. Soc. 22 (4) (2009), 963-993.

[2] J.-P. Cahen, J.-L. Chabert, Integer-valued polynomials, Amer. Math. Soc. Surveys and Monographs, 48, Providence, 1997.

[3] S. Evrard, Y. Fares, K. Johnson. Integer valued polynomials on lower triangular integer matrices, Monats. für Math. 170 (2013), no. 2, 147-160.

[4] S. Frisch. Polynomial Separation of Points in Algebras, S. Chapman (ed.), Arithmetical Properties of Commutative Rings and Modules (Chapel Hill Conf.), Dekker 2005, pp. 249-254.

[5] S. Frisch. Integer-valued polynomials on algebras, J. of Algebra 373 (2013), 414-425. 
[6] R. Gilmer. Contracted ideals with respect to integral extensions. Duke Math. J. 34 (1967), 561-571.

[7] K. Alan Loper, Nicholas J. Werner. Generalized Rings of Integer-valued Polynomials, J. Number Theory 132 (2012), no. 11, 2481-2490.

[8] N. H. McCoy. Concerning matrices with elements in a commutative ring, Bull. Amer. Math. Soc. 45 (1939), no.4, 280-284.

[9] G. Peruginelli. Integral-valued polynomials over sets of algebraic integers of bounded degree, submitted, http://arxiv.org/abs/1301.2045 